\title{
Considerações sobre a resistência mecânica e o processo de hidratação de cimentos supersulfatados (CSS) formulados com fosfogesso
}

\section{Considerations on the mechanical behavior and hydration process supersulfated cement (CSS) formulated with phosphogypsum}

\footnotetext{
${ }^{1}$ Programa de Pós-graduação em Tecnologia de Processos Químicos e Bioquímicos - PPGTP/UTFPR CP: 571- 85503390, Pato Branco, PR e-mail: gracioli@alunos,utfpr,edu,br ${ }^{2}$ Departamento de Engenharia Civil - UTFPR CP: 571- 85503-390, Pato Branco, PR e-mail: maxwell@alunos.utfpr.edu.br; cheila.beutler@gmail.com; andreza2694@hotmail.com; ilofilho@yahoo.com.br; angulski@utfpr.edu.br
}

\begin{abstract}
RESUMO
O Cimento supersulfatado (CSS) é conhecido por ser um aglomerante de baixo impacto ambiental devido aos materiais que o compõem. Os principais constituintes do CSS são escória de alto forno (até 90\%), sulfato de cálcio (10-20\%) e uma pequena quantidade de ativador alcalino (até 5\%). Estudos destacam a substituição da fonte de sulfato de cálcio natural, gipsita, por uma fonte alternativa, como o fosfogesso. No Brasil, a produção deste material é cerca de 4,5 milhões de toneladas por ano. Pelo fato do CSS compreender um maior teor de sulfato de cálcio $(20 \%)$ em sua composição, em relação ao cimento Portlant, permite um maior consumo de fosfogesso. Esta pesquisa aborda o uso do fosfogesso como fonte alternativa de sulfato de cálcio na elaboração de CSS, a fim de relatar os efeitos que diferentes teores de sulfato de cálcio (10 e 20\%) e de ativador alcalino $(0,2,0,5$ e $0,8 \%)$ exercem no desempenho deste tipo de cimento, bem como na formação de compostos hidratados. A análise dos resultados mostrou que CSS feito com fosfogesso atendeu aos requisitos mínimos de resistência à compressão exigidos pela norma europeia para cimentos supersulfatados (EN 15743/2010). Foram observadas baixas taxas de calor de hidratação, as quais foram influenciadas principalmente pelo teor de ativador alcalino. $\mathrm{O}$ excesso de ativador alcalino $(\mathrm{KOH})$ teve implicações distintas nas pastas com diferentes percentuais de sulfato de cálcio. Naquelas com baixo teor $(10 \%), 0,8 \%$ de KOH implicou queda de resistência, enquanto que naquelas com alto teor de sulfato de cálcio (20\%), o alto ter de $\mathrm{KOH}$ implicou a instabilidade da etringita.
\end{abstract}

Palavras-chave: cimento supersulfatado, fosfogesso, sulfato de cálcio, ativador alcalino, etringita.

\section{ABSTRACT}

Supersulfated Cements (SSC) are composed from blast furnace slag (90\%), calcium sulfate (10-20\%) and a small amount of alkali activator (up 5\%). Gypsum is a conventional source of calcium sulfate, however, it can be replaced by phosphogypsum (PG), a byproduct from the production of phosphoric acid $\left(\mathrm{H}_{3} \mathrm{PO}_{4}\right)$ with similar chemical and mineralogical composition of the gypsum. In Brazil, the production of this material is about 4.5 million tons per year. Because the SSC contains a higher calcium sulfate content (20\%) in relation to Portland cement, a higher consumption of phosphogypsum is possible. The goal of this study was to investigate the phosphogypsum (PG) as an alternative source of calcium sulfate in order to obtain CSS. In addition to use of PG, the effects of both calcium sulfate and alkali activator content on the process of hydration were investigated. The results showed that SSC made with phosphogypsum met the minimum compressive strength required by the European standard for SSC (EN 15743/2010). Low heat of hydration rates mainly influenced by the low alkali activator content was observed. The excess of alkali activator $(\mathrm{KOH})$ had differ- 
ent influences according to calcium sulfate content. In pastes made with low content $(10 \%), 0.8 \%$ of $\mathrm{KOH}$ reduced the compressive strength, while in those with a high calcium sulfate content $(20 \%)$ the high alkaline content resulted in the instability of ettringite.

Keywords: supersulfated cement, phosphogypsum, calcium sulfate, alkaline activator, ettringite.

\section{INTRODUÇÃO}

Nos últimos anos, grande atenção tem sido dada ao desenvolvimento de cimentos especiais, capazes de reduzir as emissões de $\mathrm{CO}_{2}$, energia e consumo de calcário.

Cimentos Supersulfatados (CSS) são aglomerantes isentos (ou quase) de clínquer, compostos por escória de alto forno, $(80-85 \%)$, sulfato de cálcio (10-20\%) e uma pequena quantidade de ativador alcalino, normalmente Cimento Portland ou hidróxidos [1-3].

A ausência de clínquer (ou teores muito baixos) em CSS reduz de forma significativa o impacto ambiental envolvido na sua produção, no que se refere principalmente ao menor consumo de energia e menor emissão de $\mathrm{CO}_{2}$ em relação ao cimento Portland. Além das questões ambientais, CSS proporcionam maior resistência ao ataque de sulfato e menor calor de hidratação. [1,4-7].

A ativação de escória granulada de alto-forno por sulfatos foi documentada pela primeira vez por Hans Kühl em 1908 [5] e décadas depois resultou na sua padronização na Alemanha através da norma DIN 4210 em 1970 [8]. Mais tarde, mudanças nos processos de fabricação de ferro gusa causaram alterações nas características das escórias geradas, as quais deixaram de cumprir o requisito mínimo de $13 \%$ de teor de $\mathrm{Al}_{2} \mathrm{O}_{3}$. As escórias passaram então a ser empregadas como adição em cimentos Portland [1].

Recentemente na Europa, os cimentos supersulfatados tiveram sua norma atualizada pela EN 15743/2010 [9], a qual deixou de exigir um teor mínimo de $\mathrm{Al}_{2} \mathrm{O}_{3}$ para as escórias, como no passado. Recentes pesquisas também têm mostrado a possibilidade de obter cimentos supersulfatados a partir de escórias com baixo teor de alumina [10-12]. Outra vantagem do CSS em relação ao cimento Portland, é o fato deste aglomerante possuir maior teor de sulfato de cálcio em sua formulação, de 10 a $20 \%$, o que possibilita valorizar fontes alternativas, como o fosfogesso.

O fosfogesso é um subproduto industrial, proveniente da indústria de fertilizantes, gerado a partir da produção de ácido fosfórico $\left(\mathrm{H}_{3} \mathrm{PO}_{4}\right)$. Este processo ocorre a partir de uma rocha primária fosfática que reage com o ácido sulfúrico resultando na formação de um precipitado de sulfato de cálcio, chamado fosfogesso [13-15]. Neste processo, o ácido sulfúrico dissolve a rocha, obtendo-se o ácido fosfórico, fosfogesso e ácido fluorídrico, representado pela Equação 1 [16,17].

$$
\mathrm{Ca}_{5}\left(\mathrm{PO}_{4}\right)_{3} \mathrm{~F}+5 \mathrm{H}_{2} \mathrm{SO}_{4}+10 \mathrm{H}_{2} \mathrm{O} \rightarrow 3 \mathrm{H}_{3} \mathrm{PO}_{4}+5 \mathrm{CaSO}_{4} \cdot 2 \mathrm{H}_{2} \mathrm{O}+\mathrm{HF}
$$

Em CSS, a fonte de sulfato de cálcio pode ser inserida na fase di-hidratada $\left(\mathrm{CaSO}_{4} \cdot 2 \mathrm{H}_{2} \mathrm{O}\right)$, como o fosfogesso, a hemihidratada $\left(\mathrm{CaSO}_{4}, 0,5 \mathrm{H}_{2} \mathrm{O}\right)$ e anidra $\left(\mathrm{CaSO}_{4}\right)$, chamada de anidrita, sendo a última mais comum [15]. Para tanto, é necessário a calcinação da fonte de sulfato de cálcio, a fim de eliminar as moléculas de água presentes na composição, obtendo-se a anidrita [18-22].

A viabilidade do uso de fosfogesso em substituição à fonte convencional de sulfato de cálcio em CSS vem sendo verificada por vários estudos [20,23-25]. Recentemente, o desempenho do CSS elaborado com anidrita proveniente do fosfogesso foi comparado com amostras confeccionadas com a fonte convencional. Nestes estudos, os resultados de resistência à compressão para o CSS contendo fosfogesso foram equivalentes e em algumas amostras superiores ao CSS elaborado com a fonte convencional [26, 27].

Em cimentos supersulfatados, a reação do sulfato de cálcio com a escória pode ser simplificada pela Equação $2^{1}$. O ativador alcalino, neste caso, cimento Portland, inicialmente hidrata, formando o hidróxido de cálcio $(\mathrm{CH})$, o qual aumenta o $\mathrm{pH}$ do sistema e promove a dissolução da escória $\left(\mathrm{C}_{5} \mathrm{~S}_{3} \mathrm{~A}\right)$. Os íons de alumínio, cálcio e silício dissolvidos reagem com o sulfato de cálcio adicionado $(\mathrm{C} \overline{\mathrm{S}})$ para formar inicialmente etringita $\left(\mathrm{C}_{6} \mathrm{AS}_{3} \mathrm{H}_{32}\right)$ e, de forma mais lenta, o silicato de cálcio hidratado (CSH) [3].

$$
\mathrm{C}_{5} \mathrm{~S}_{3} \mathrm{~A}+\mathrm{CH}+3 \mathrm{C} \overline{\mathrm{S}}+34 \mathrm{H} \rightarrow \mathrm{C}_{6} \mathrm{AS}_{3} \mathrm{H}_{32}+3 \mathrm{CSH}
$$

\footnotetext{
${ }^{1} \mathrm{C}_{5} \mathrm{~S}_{3} \mathrm{~A}=\mathrm{Ca}_{2} \mathrm{Al}_{2} \mathrm{Si}_{2} \mathrm{O}_{7}, \mathrm{CH}=\mathrm{Ca}(\mathrm{OH})_{2}, \mathrm{CS}=\mathrm{CaSO}_{4}, \mathrm{H}=\mathrm{H}_{2} \mathrm{O}, \mathrm{C}_{6} \mathrm{AS}_{3} \mathrm{H}_{32}=\mathrm{Ca}_{6} \mathrm{Al}_{2}(\mathrm{OH})_{12}\left(\mathrm{SO}_{4}\right)_{3} \cdot 26 \mathrm{H}_{2} \mathrm{O}$,

$\mathrm{C}-\mathrm{S}-\mathrm{H}=\mathrm{Ca}_{3} \mathrm{H}_{2} \mathrm{SiO}_{6}, \mathrm{C}_{6} \mathrm{~A} \overline{\mathrm{S}} \mathrm{H}_{12}=3 \mathrm{CaO} \cdot 3 \mathrm{CaSO}_{4} \cdot \mathrm{Al}_{2} \mathrm{O}_{3} \cdot 12 \mathrm{H}_{2} \mathrm{O}$
} 
Na ausência de sulfato de cálcio, os íons disponíveis de cálcio e alumínio podem reagir com a etringita formada na Equação 2, e convertê-la em monossulfato, conforme Equação 3 [20].

$$
6 \mathrm{Ca}^{2+}+\mathrm{Al}^{3+}+0.5 \mathrm{C}_{6} \mathrm{AS}_{3} \mathrm{H}_{32}+2 \mathrm{H} \rightarrow 1.5 \mathrm{C}_{6} \mathrm{AS}_{12}
$$

Ao contrário, altos teores de sulfato de cálcio podem converter a anidrita residual $(C \bar{S})$ em gipsita secundária $\left(C \bar{S} H_{2}\right)$, conforme Equação 4, gerando o aparecimento de fissuras devidas à pressão nos vazios e queda de resistência mecânica como principal consequência [20,22].

$$
\mathrm{C} \overline{\mathrm{S}}+2 \mathrm{H} \rightarrow \mathrm{C} \overline{\mathrm{S}} \mathrm{H}_{2}
$$

O teor de ativador alcalino também exerce papel importante no processo de hidratação e tem sido estudado em alguns trabalhos recentes. Segundo Mun et al [20], o teor de ativador alcalino em excesso, pode provocar a instabilidade da etringita se o $\mathrm{pH}$ alcançar valores próximos de 13, com consequente queda de resistência. Num outro estudo, Matshei et al [28] atribuíram o atraso nos tempos de pega e a baixa resistência mecânica também ao excesso de ativador. Porém, os autores relacionaram este comportamento à grande disponibilidade de íons sulfato, propiciada pela maior concentração de álcalis na solução. Os autores explicam que a grande concentração de íons sulfato implicou a precipitação e crescimento de cristais de etringita na superfície dos grãos de escória, causando seu isolamento e inibindo posterior hidratação.

Desta forma, além da dosagem de sulfato de cálcio, aquela referente ao ativador é essencial para garantir a dissolução da escória, formação de produtos hidratados e estabilidade dos mesmos. Entretanto, as implicações quanto à variação do teor de sulfato de cálcio e de ativador alcalino no desempenho dos cimentos supersulfatados são pouco estudadas, e portanto, poucos dados estão disponíveis na bibliografia.

Este artigo visa avaliar o desempenho de cimentos supersulfatados obtidos a partir do fosfogesso, formulados com variações dos teores de sulfato de cálcio e ativador alcalino, a fim de contribuir no entendimento do seu processo de hidratação o qual é ainda pouco conhecido. Os objetivos específicos foram:

- avaliar a adequabilidade do fosfogesso como fonte de sulfato de cálcio em CSS;

- identificar os teores ótimos de ativador alcalino/sulfato de cálcio/escória no comportamento do CSS;

- contribuir no entendimento do papel do teor de sulfato de cálcio e de ativador alcalino no desempenho mecânico e processo de hidratação do CSS.

\section{MATERIAIS E MÉTODOS}

Para avaliação do cimento supersulfatado (CSS) obtido com fosfogesso, incialmente fez a caracterização dos materiais (fosfogesso e escória) além da definição dos teores de cada um deles nas formulações estudadas. A partir disto, o CSS foi avaliado em testes de resistência à compressão e o processo de hidratação foi investigado por testes de calorimetria, difração aos raios X (DX), análise térmica por calorimetria exploratória por condução (DSC) e por microscopia eletrônica de varredura (MEV).

\subsection{Materiais}

Para a elaboração das argamassas e pastas de CSS utilizou-se em sua composição escória de alto forno, fosfogesso como fonte de sulfato de cálcio e $\mathrm{KOH}$ como ativador alcalino.

A escória de alto-forno gerada por uma empresa brasileira utilizando o carvão mineral como combustível. A mesma foi triturada em moinho de bolas por um período padrão de 2 horas (Figura 1). A Tabela 1 apresenta a composição química da escória, obtida por fluorescência de raios X (FRX). A escória utilizada atendeu aos limites impostos pela EN 15743 para sua utilização em CSS.

A fim de caracterizar a escória de alto forno utilizada nas amostras foram realizadas análise de granulometria a laser e difração de raios X (DRX), apresentada na Figura 1. A granulometria apresentou um tamanho médio de partícula de $25,25 \mu \mathrm{m}$. Na análise de DRX não foram identificados picos significativos para a escória utilizada, apontando uma característica amorfa para a mesma. 
Tabela 1: Composição química (\%) da escória de alto forno por FRX.

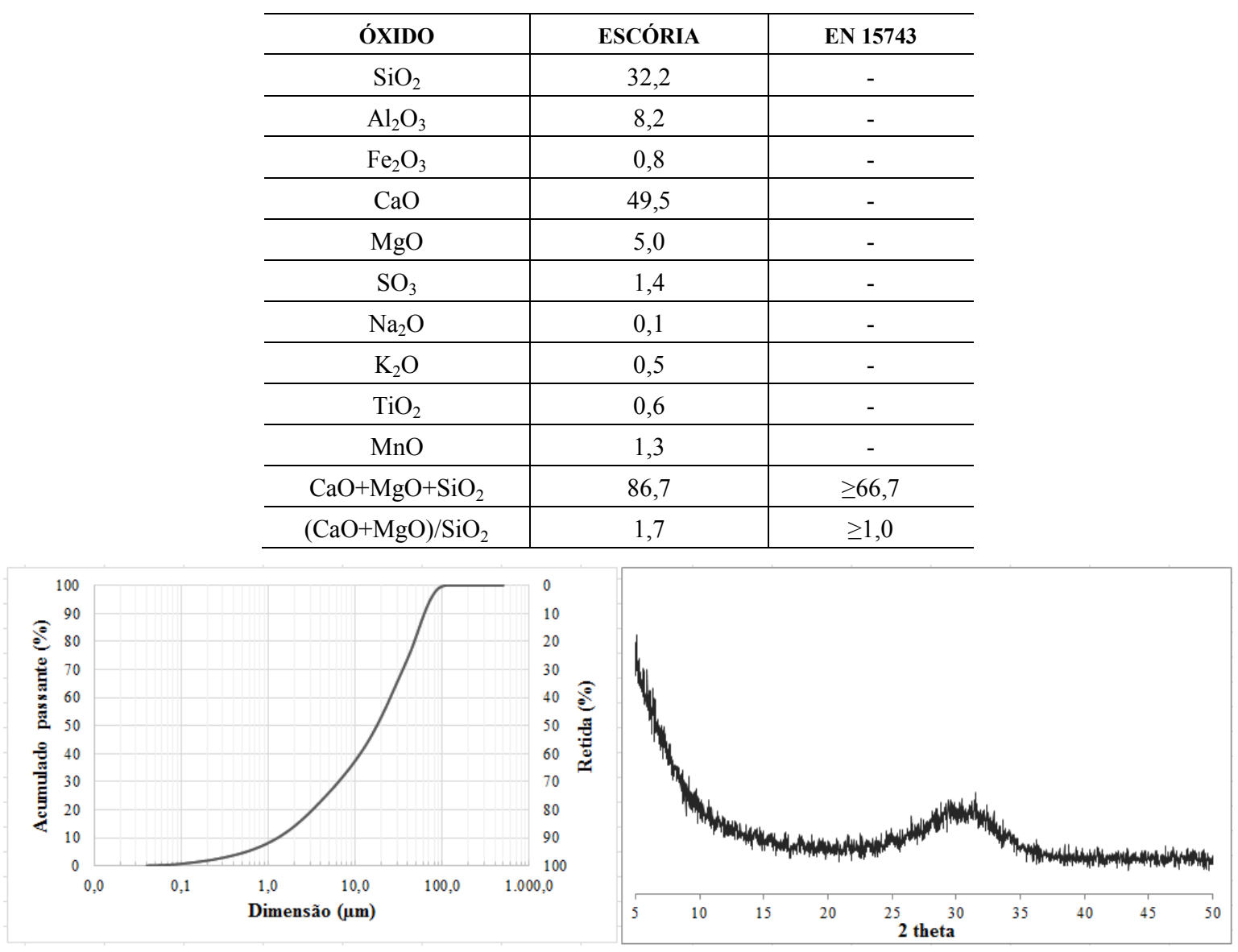

Figura 1: Granulometria a laser e análise de DRX da escória de alto forno.

O fosfogesso empregado como fonte de sulfato de cálcio é proveniente da indústria brasileira. A composição química deste material também foi analisada por FRX, representada na Tabela 2. É possível notar a presença dos principais componentes $\left(\mathrm{SO}_{3}\right.$ e $\left.\mathrm{CaO}\right)$ em maior percentual, como esperado. Para a obtenção da sua forma anidra, o fosfogesso foi passado em peneira de $0,150 \mathrm{~mm}$ e em seguida calcinado em mufla, em temperatura de $650{ }^{\circ} \mathrm{C}$, durante 1 hora, a uma taxa de aquecimento de $50{ }^{\circ} \mathrm{C} \mathrm{min}{ }^{-1}$, conforme procedimento adotado por Angulski da Luz e Hooton [10] (Figura 2). Além disso, foi realizada a medição do potencial hidrogeniônico $(\mathrm{pH})$ do fosfogesso di-hidratado e anidro (anidrita), os quais apresentaram valores de 5,7 e 7,1 respectivamente.

A análise de DRX também foi realiza para o fosfogesso in natura e calcinado (Figura 3). Através do difratograma do fosfogesso in natura $\left(\mathrm{CaSO}_{4} \cdot 2 \mathrm{H}_{2} \mathrm{O}\right)$ notam-se picos característicos apenas de gipsita e, após a calcinação do fosfogesso os picos formados são de anidrita $\left(\mathrm{CaSO}_{4}\right)$. Isto comprova a eficácia da calcinação tornando o fosfogesso di-hidratado para anidro.

Tabela 2: Composição química (\%) do fosfogesso por FRX.

\begin{tabular}{c|c}
\hline ÓXIDO & FOSFOGESSO \\
\hline $\mathrm{SO}_{3}$ & 46,8 \\
\hline $\mathrm{CaO}$ & 38,5 \\
\hline $\mathrm{SiO}_{2}$ & 1 \\
\hline $\mathrm{Al}_{2} \mathrm{O}_{3}$ & 0,3 \\
\hline $\mathrm{Fe}_{2} \mathrm{O}_{3}$ & 0,2 \\
\hline $\mathrm{P}_{2} \mathrm{O}_{5}$ & -- \\
\hline $\mathrm{MgO}$ & 0,2 \\
\hline
\end{tabular}




\begin{tabular}{c|c}
\hline $\mathrm{SrO}$ & 0,1 \\
\hline $\mathrm{TiO}_{2}$ & -- \\
\hline $\mathrm{K}_{2} \mathrm{O}$ & 0,1 \\
\hline $\mathrm{MnO}$ & $<0,1$ \\
\hline $\mathrm{BaO}$ & -- \\
\hline $\mathrm{CeO}_{2}$ & -- \\
\hline $\mathrm{F}$ & -- \\
\hline Perda ao fogo & 12,82 \\
\hline
\end{tabular}

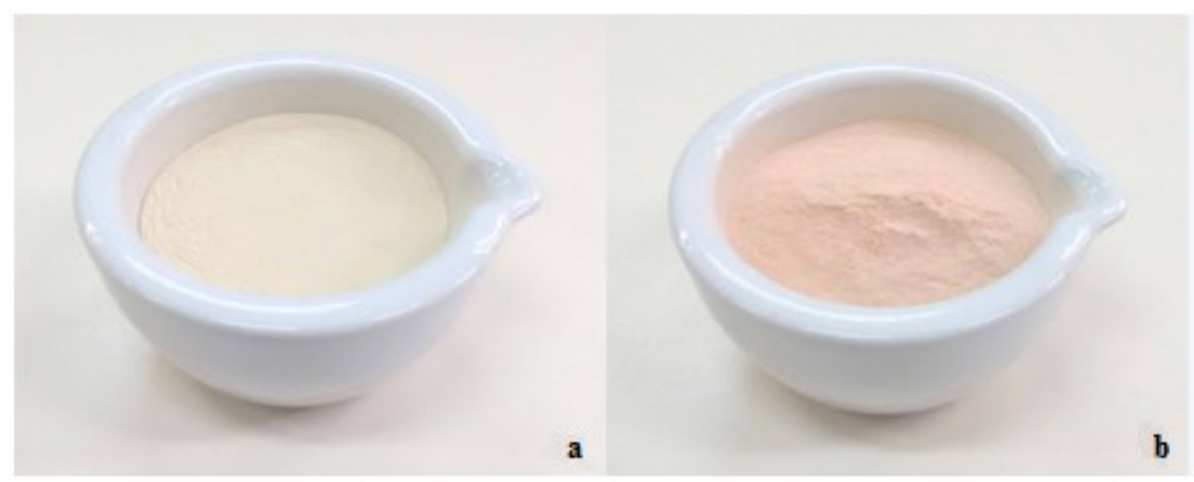

Figura 2: Fosfogesso a) di-hidratado (in natura) e b) anidrita (calcinado).

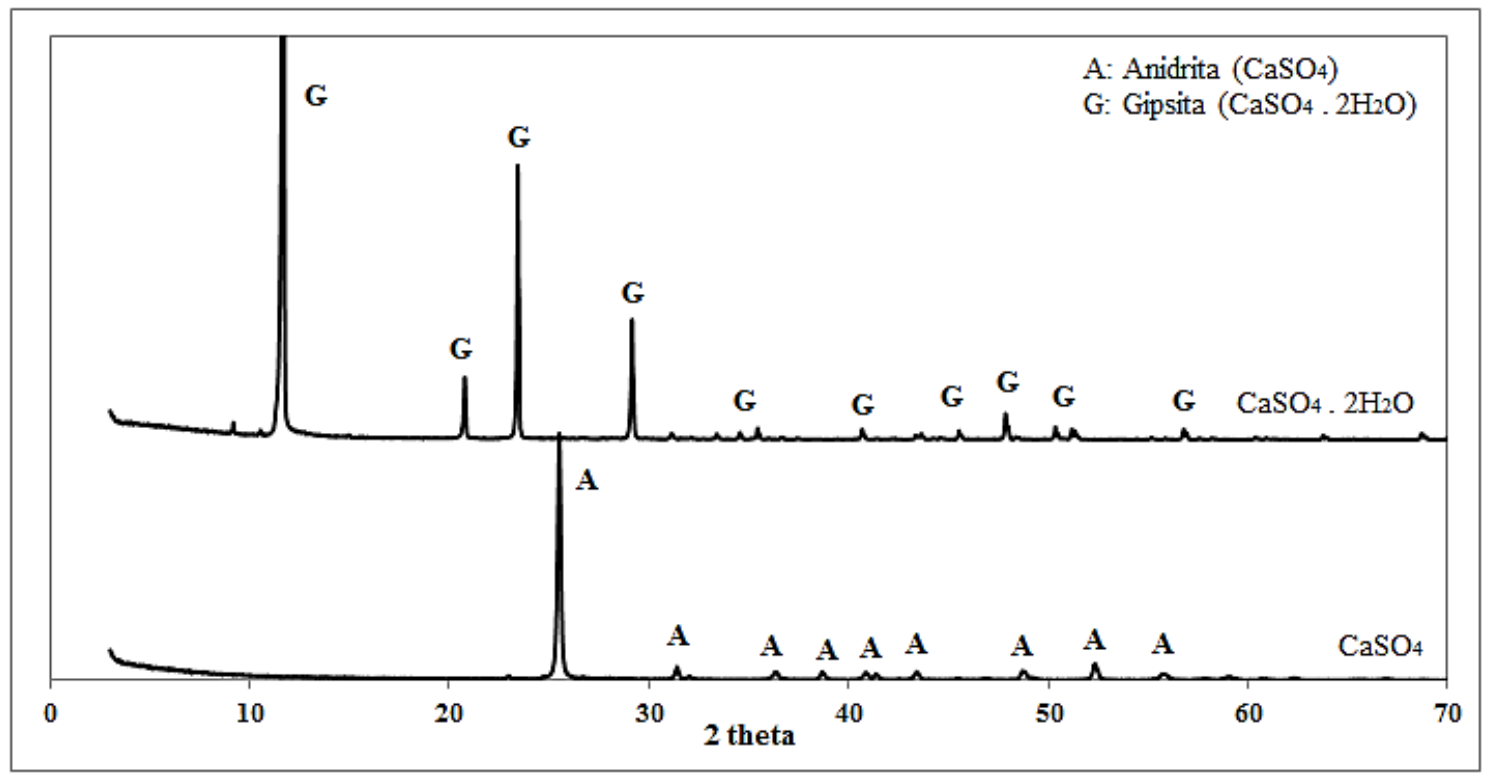

Figura 3: Fosfogesso in natura $\left(\mathrm{CaSO}_{4} \cdot 2 \mathrm{H}_{2} \mathrm{O}\right)$ e após calcinação $\left(\mathrm{CaSO}_{4}\right)$.

\subsection{Métodos}

Na elaboração das amostras de CSS (argamassas e pastas) optou-se pela utilização de duas proporções de escória ( 89 e $90 \%$ ) e fosfogesso (10 e $20 \%$ ), sendo que estas assumiram três variações do percentual de ativador alcalino $(\mathrm{KOH})$, de $0,2,0,5$ e $0,8 \%$, conforme Tabela 3. Os percentuais visaram cobrir os valores máximos e mínimos de cada material (sulfato de cálcio, escória e ativador alcalino) encontrados na literatura. 
Tabela 3: Proporção (\%) dos materiais utilizados na composição do CSS.

\begin{tabular}{c|c|c|c}
\hline AMOSTRA & ESCÓRIA & FOSFOGESSO & KOH \\
\hline \multirow{2}{*}{80.20} & \multirow{3}{*}{80} & \multirow{2}{*}{20} & 0,2 \\
\cline { 3 - 4 } & & & 0,5 \\
\hline \multirow{2}{*}{90.10} & \multirow{2}{*}{20} & \multirow{2}{*}{10} & 0,8 \\
\hline & & & 0,2 \\
\hline
\end{tabular}

Para a realização da resistência à compressão, as argamassas foram preparadas na proporção 1: 2,75: 0,485 (CSS: areia: água), de acordo com a ASTM C109 [29], moldadas em fôrmas prismáticas $4 \mathrm{~cm} \times 4 \mathrm{~cm} x 16 \mathrm{~cm}$, conforme a EN 15743 [9] e mantidas em câmera úmida (UR $>95 \%$ e $\mathrm{T}=23^{\circ} \mathrm{C}$ ) por 24 horas, até serem desmoldadas e imersas em solução de água de cal saturada na quantidade de $3 \mathrm{~g} / \mathrm{L}$ até completarem as idades de teste de 7 e 28 dias.

Para a medição do calor de hidratação, as pastas de CSS foram preparadas com uma relação a/c de 0,4. Usando um calorímetro isotérmico (Calmetrix), as medições de libertação de calor foram realizadas usando $40 \mathrm{~g}$ de pasta, por um período de 7 dias, à $23^{\circ} \mathrm{C}$.

Para as análises mineralógicas, foram utilizadas $20 \mathrm{~g}$ de pasta $(\mathrm{a} / \mathrm{c}$ de 0,4$)$ as quais foram mantidas em câmara úmida por $48 \mathrm{~h}$, e após imersas em água de cal saturada $(3 \mathrm{~g} / \mathrm{L})$ até os 7 e 28 dias. A interrupção do processo de hidratação foi realizada por troca de solvente, através de imersão em acetona por 2 horas. Em seguida, o material foi filtrado, triturado e peneirado na malha $0,150 \mathrm{~mm}$. Para as análises de difração de raios X (DRX) foi utilizada radiação CuKa, intervalo de leitura $2 \theta$ de $3^{\circ}-70^{\circ}$, passo de $0,02^{\circ}$ e tempo de contagem de $10 \mathrm{~s}$. Na análise de calorimetria exploratória por condução (DSC) os parâmetros utilizados foram atmosfera inerte de gás $\mathrm{N}_{2}$ com fluxo de $100 \mathrm{~mL} \mathrm{~min}{ }^{-1}$, taxa de aquecimento de $5{ }^{0} \mathrm{C} \mathrm{min}^{-1}$ em uma faixa de leitura de 30 a $300{ }^{\circ} \mathrm{C}$. Antes da trituração, alguns fragmentos foram separados para análise de microscopia eletrônica de varredura (MEV), os quais foram submetidos à metalização com ouro. Além da identificação dos compostos formados, o uso do MEV como técnica de investigação visou avaliar detalhes da morfologia destes e eventual presença de microfissuras e foram realizadas nas amostras de CSS que apresentaram melhor desempenho mecânico nos grupos com diferentes teores de sulfato de cálcio. Todas os parâmetros das análises descritos anteriormente foram baseados no trabalho de Angulski da Luz e Hooton [10].

\section{RESULTADOS E DISCUSSÃO}

Os resultados a seguir apresentam o comportamento apresentado pelos cimentos supersulfatados nos testes de resistência à compressão, de calorimetria e na investigação da microestrutura (DRS, DSC e MEV).

\subsection{Resistência à compressão}

Os testes de resistência à compressão dos cimentos supersulfatados formulados com 10 e $20 \%$ de sulfato de cálcio, 90_10 e 80_20 respectivamente, e variação do ativador alcalino de $0,2,0,5$ e $0,8 \%$, aos 7 e 28 dias, estão apresentados na Figura 4.

Em relação ao percentual de sulfato de cálcio nota-se que os valores mais altos são observados nas amostras com $10 \%$ de fosfogesso, tanto aos 7 quanto aos 28 dias de cura, com exceção das amostras com $0,2 \%$ de ativador alcalino.

O percentual de ativador influenciou de maneira diferente as amostras conforme o teor de fosfogesso utilizado. Quando se fez o uso de um menor teor de fosfogesso (10\%) o melhor desempenho foi apresentado nas amostras com 0,5 e 0,8\% de ativador. Quando utilizado o maior percentual de fosfogesso (20\%) o melhor resultado de resistência à compressão foi observado apenas na amostra com menor teor de ativador $(0,2 \%)$. Isso indica que um menor teor de fosfogesso (10\%) implica maiores quantidades de ativador alcalino necessário à dissolução da escória, a qual está presente nestas argamassas em maior quantidade (90\%). 


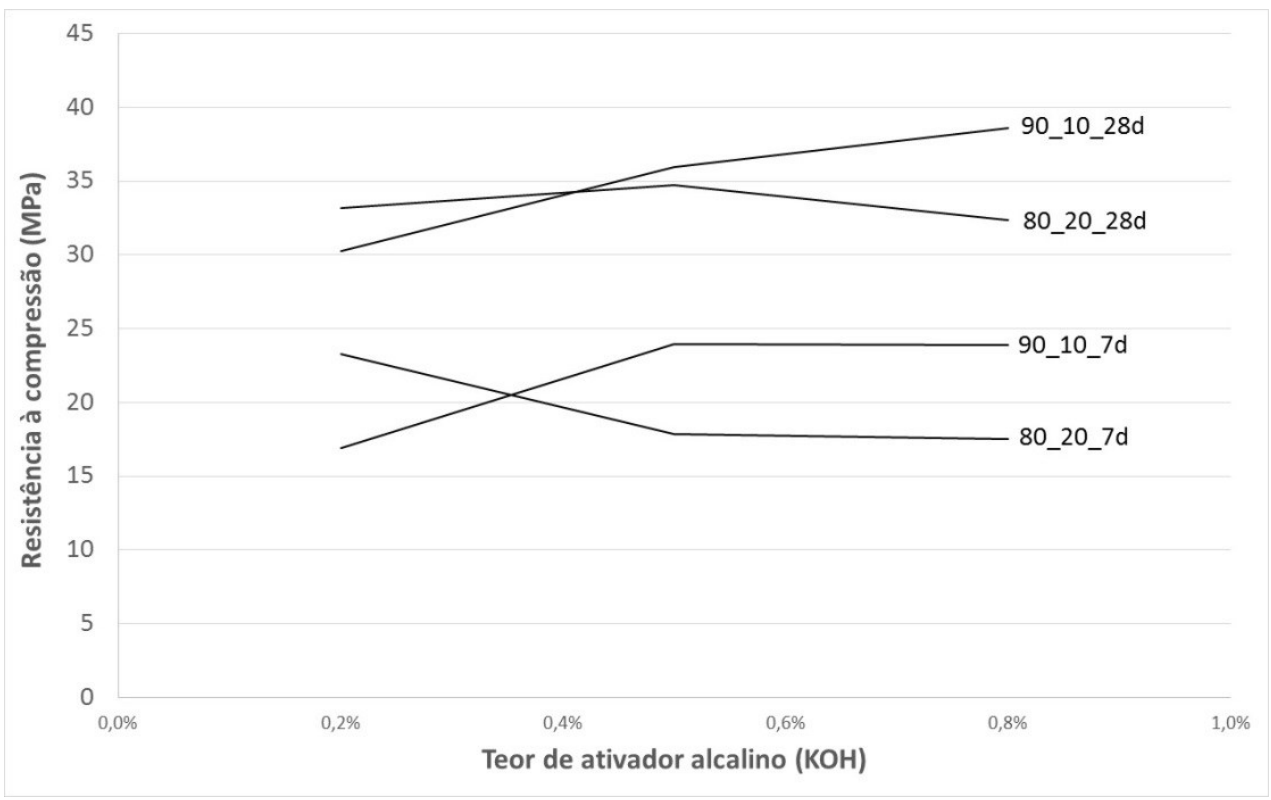

Figura 4: Resistência à compressão das amostras de CSS com 20\%(80_20) e 10\% (90_10) de fosfogesso com 0,2, 0,5 e $0,8 \%$ de ativador alcalino aos 7 e 28 dias.

De acordo com a EN 15743/2010, todos os resultados de resistência à compressão atendem aos valores exigidos pela normativa. Nas duas idades avaliadas de 7 e 28 dias de hidratação, as amostras de CSS elaboradas atendem aos valores exigidos pela classe 32,5 N (7 dias $\geq 16,0$ e 28 dias $\geq 32,5 \leq 52,5$ ), com exceção da amostra com $10 \%$ de sulfato de cálcio (90_10) com $0,2 \%$ de ativador, que atingiu 30,3 MPa aos 28 dias de hidratação.

\subsection{Calorimetria}

A Figura 5 apresenta as curvas de calorimetria referentes à taxa e ao calor total liberado, nas amostras de cimento supersulfatados (CSS), até os 7 dias. 


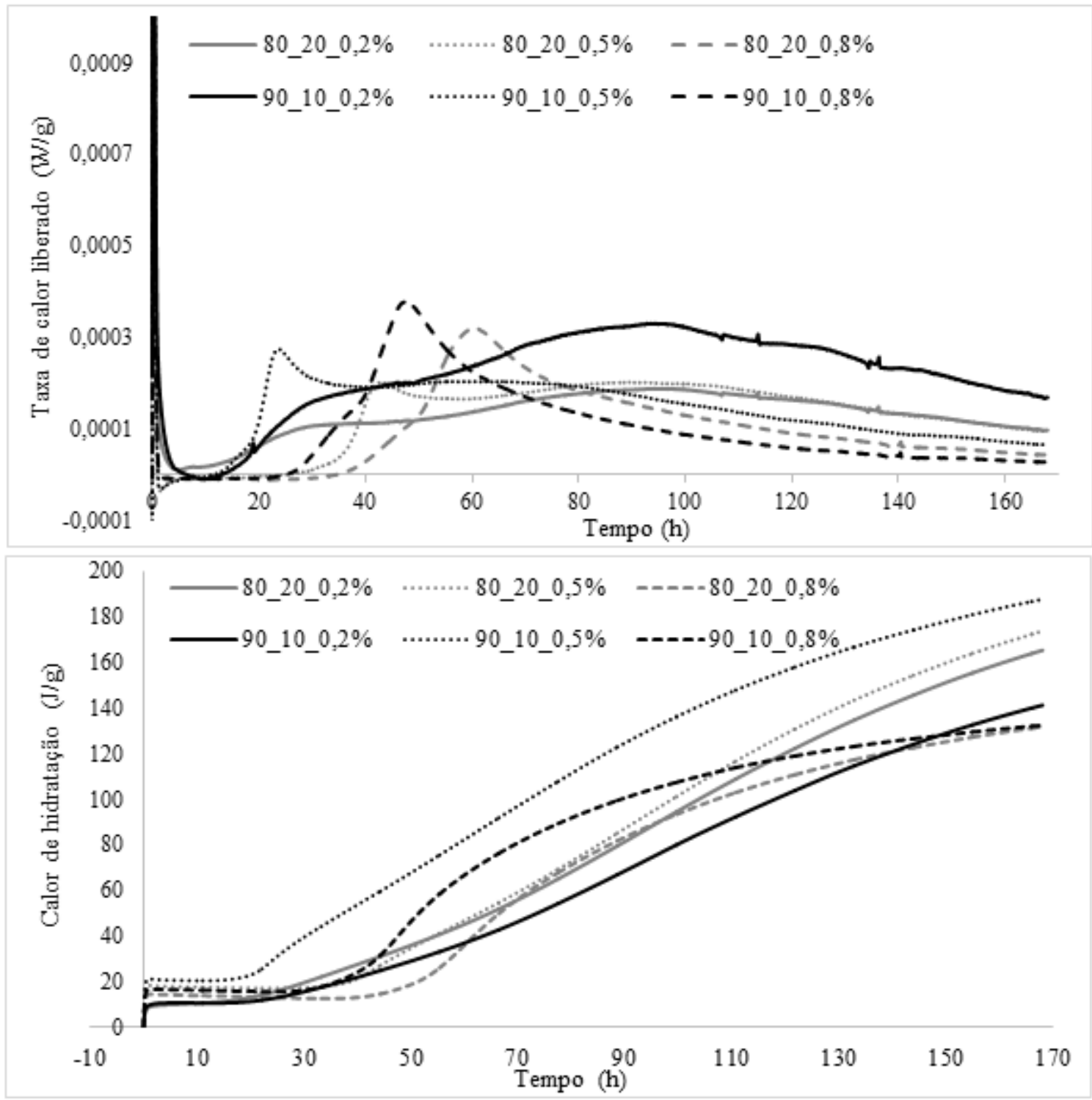

Figura 5: Curvas de calorimetrias: taxa e calor total liberado nas amostras de CSS com 20\% (80_20) e 10\% (90_10) de fosfogesso contendo $0,2,0,5$ e $0,8 \%$ de ativador alcalino.

É possível notar que a similaridade entre as curvas de taxa de calor liberado é mais influenciada pelo teor de ativador alcalino do que pelo teor de fosfogesso. As amostras com $0,5 \%$ de ativador apresentam picos elevados nas primeiras horas de hidratação (aproximadamente até 48 horas), as amostras com $0,8 \%$ ativador apresentam os mesmos picos, em maior intensidade, até 72 horas, caracterizando reações de hidratação mais intensas. Já os CSS's com $0,2 \%$ de ativador alcalino não apresentam pico de hidratação evidente, caracterizando reações de hidratação bastante lentas. A hipótese levantada por Matschei et al. [28] de que o alto teor de sulfato de cálcio, associado ao alto teor de ativador alcalino poderia causar aumento nos tempos de pega, pode justificar o comportamento das pastas devido à precipitação e crescimento de cristais de etringita na superfície dos grãos de escória isolando-a. Desta forma, pode-se justificar o maior período dormente observado nas pastas com $0,8 \%$ de ativador, em relação à $0,5 \%$ e também naquelas com $20 \%$ de fosfogesso (fonte de sulfato) em relação à $10 \%$ de fosfogesso.

Em relação ao gráfico de calor acumulado, observa-se também que o teor de ativador exerceu forte influência no traçado das curvas. Observa-se que as pastas com $0,5 \%$ de ativador apresentaram os maiores valores de calor acumulado, apontando ser este o teor que propicia mais reações de hidratação, para ambos os teores de sulfato de cálcio. Ao fim dos 7 dias (168) percebe-se que a influência do teor de sulfato de cálcio no calor acumulado tende a ser reduzida conforme o teor de ativador alcalino é aumentado. 


\subsection{Análises mineralógicas}

As análises a seguir apresentam a investigação da microestrutura das pastas de CSS estudadas, através das técnicas de difração aos raios X (DX), análise térmica por calorimetria exploratória por condução (DSC) e por microscopia eletrônica de varredura (MEV).

\subsubsection{Difração aos Raios X (DRX)}

A Figura 6 apresenta os gráficos referentes às análises de DRX realizadas aos 7 e 28 dias.

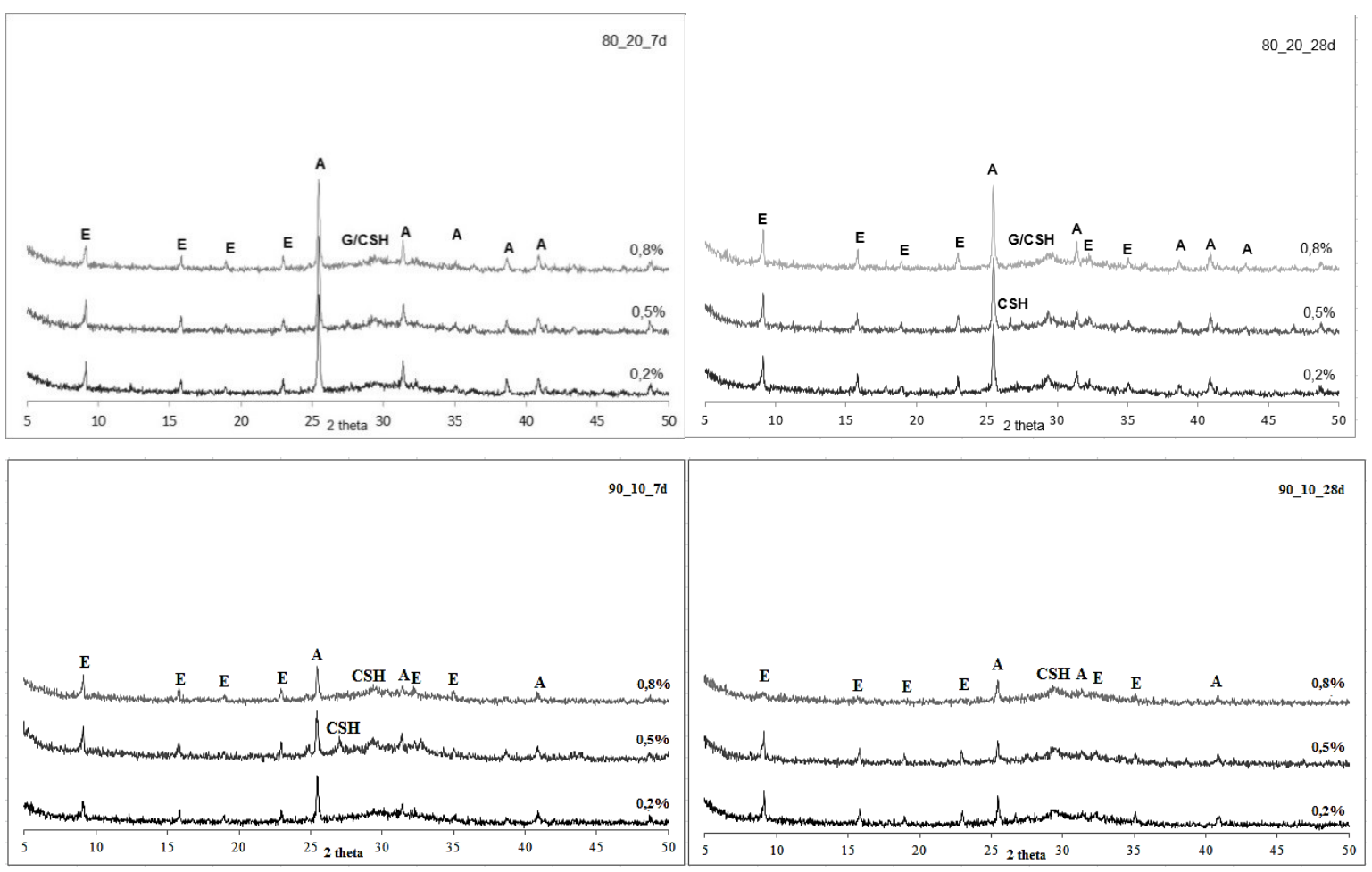

Figura 6: Análise de DRX das amostras com 20\% (80_20) e 10\% (90_10) de fosfogesso com 0,2, 0,5 e 0,8\% de ativador alcalino aos 7 e 28 dias (A: anidrita; $\mathrm{CSH}$ : silicato de cálcio hidratado; E: etringita).

Em relação ao consumo de sulfato de cálcio, ambas as formulações, 10 e $20 \%$ de fosfogesso, $90 \_10$ e $80 \_20$, respectivamente, apresentaram um consumo gradativo de anidrita dos 7 para os 28 dias de hidratação para a formação de etringita. Também em ambas as formulações, nos três teores de ativador $(0,2,0,5$ e $0,8 \%$ ), o pico de anidrita ainda se mantém, mostrando que, mesmo aos 28 dias, a anidrita se encontra disponível no sistema. As amostras com $20 \%$ de fosfogesso (80_20), por consequência da maior quantidade de sulfato de cálcio, apresentaram picos mais intensos referentes à anidrita.

Em relação à formação de etringita, a influência do teor de sulfato de cálcio acontece de forma distinta. Para as amostras com 10\% de fosfogesso, a formação ocorre majoritariamente aos 7 dias e, aos 28 dias, parece ocorrer uma redução quando o teor de ativador é de $0,8 \%$. Para as pastas com $20 \%$, a formação de etringita é mais intensa aos 28 dias e ligeiramente maior com $0,8 \%$ de ativador. Este comportamento é condizente com o aquele observado nas curvas de calorimetria, onde constatou-se que o maior teor de sulfato de cálcio e de ativador alcalino desacelera a hidratação das partículas de escória, apontado por Matshei et al [28]. Já, a redução do pico de etringita nas pastas com $10 \%$ de sulfato de cálcio e $0,8 \%$ de ativador, pode estar relacionado a sua conversão em monossulfato, que ocorre em sistemas com baixa disponibilidade de sulfato de cálcio, e/ou a sua instabilidade em meios fortemente alcalinos, observados por Mun et al. [20].

Aos 28 dias, o silicato de cálcio hidratado $(\mathrm{CSH})$ parece ser identificado num pico discreto à $29^{\circ}$. Entretanto, a identificação do CSH nos difratogramas não é simples, devido a sua caraterística amorfa e, também, devido à proximidade ao pico de gipsita, comumente presente nos sistemas SSC, como também observado em trabalhos recentes $[10,11,20]$. 


\subsubsection{Calorimetria exploratória diferencial (DSC)}

As análises térmicas as quais as amostras foram submetidas (DSC) estão apresentadas na Figura 7.
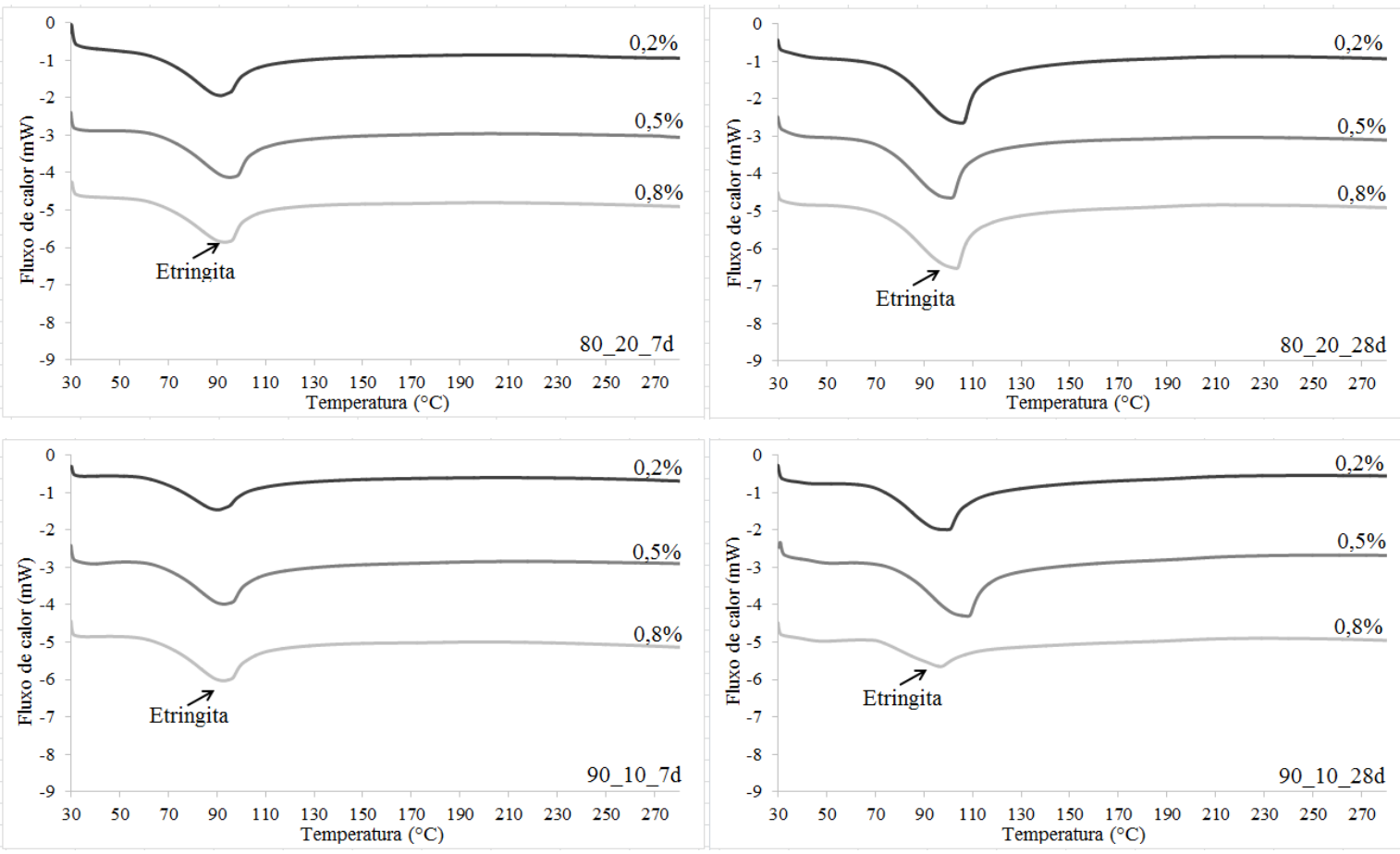

Figura 7: Análise de DSC das amostras com 20\% (80_20) e 10\% (90_10) de fosfogesso com 0,2, 0,5 e 0,8\% de ativador alcalino aos 7 e 28 dias.

É possível identificar apenas o pico referente à etringita para todas as amostras de CSS elaboradas, nas duas idades de cura. Aos 7 dias de hidratação, os teores de fosfogesso não demonstram influência significativa. Ainda nesta idade, os maiores teores de ativador alcalino $(0,5$ e $0,8 \%)$ apresentam picos de etringita mais intensos do que quando utilizado $0,2 \%$ de ativador. Isso explicaria o melhor desempenho de resistência à compressão encontrado nas amostras com 10\% de fosfogesso $\left(90 \_10\right)$ diante de maiores teores de ativador, mas são contraditórios com aqueles apresentados pelas pastas com 20\% de fosfogesso (80_20), que apresentaram resultados melhores com baixos teores de ativador. A hipótese de Matshei et al. [28] do isolamento dos grãos de escória diante de alto teor de sulfato e de álcalis é a hipótese mais aceita para estas pastas 80_20.

Aos 28 dias de cura, a presença de etringita nas pastas 80_20 e 90_10 é semelhante quando os teores de ativador são de 0,2 e $0,5 \%$, como também observado nos difratogramas (Figura 6). Entretanto, para as pastas 80_20 com a formação de etringita é ligeiramente maior com $0,8 \%$ de $\mathrm{KOH}$, e para as pastas $90 \_10$ é evidentemente menor.

De forma geral, em relação à formação da etringita, as análises de DSC confirmam as observações mencionadas nos difratogramas (Figura 6). Para as pastas com $20 \%$ de sulfato de cálcio, ocorre um aumento do pico dos 7 aos 28 dias, enquanto que para aquelas com $10 \%$ de sulfato de cálcio, a mesma tende a se manter. Nestas últimas, na pasta com $0,8 \%$ de ativador, observa-se uma pequena redução, indicando novamente uma instabilidade da etringita em meio fortemente alcalino e/ou sua conversão em monossulfato [20]. Entretanto, a presença deste composto não pode ser identificada, nem por DRX, nem por DSC.

A proximidade de ocorrência do pico de CSH da etringita nas curvas de DSC dificulta a identificação do primeiro, já que o mesmo também é de difícil identificação nas análises de DRX devido a sua baixa cristalinidade, como também verificado em trabalhos mais recentes [10,11].

\subsubsection{Microscopia eletrônica de varredura (MEV)}

Com o objetivo de visualizar os hidratos formados até os 28 dias de cura das amostras que apresentaram maior desempenho mecânico, estas foram submetidas à análise de microscopia eletrônica de varredura (MEV). A Figura 8 refere-se a amostra com $20 \%$ de fosfogesso confeccionada com 0,2 de KOH. Neste caso é possível notar grande aglomeração de etringita na superfície e a presença de CSH ao redor dos cristais, o que é espe- 
rado em sistema com baixo teor de ativador alcalino [28] e, assim, uma maior resistência mecânica, conforme foi também constatado neste estudo. Percebe-se também que muitas agulhas apresentam comprimento de até $10 \mu \mathrm{m}$

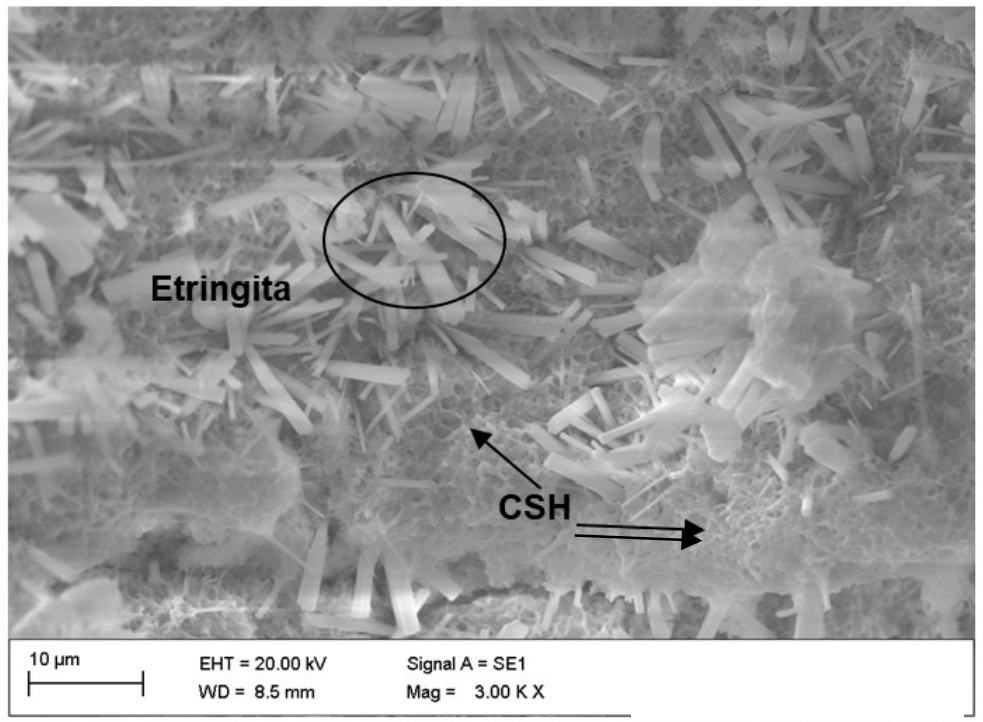

Figura 8: Microscopia eletrônica de varredura (MEV): Pasta $80 \_20$ com 0,2\% de ativador, aos 28 dias

Já nas imagens da Figura 9, referente à amostra com $10 \%$ de fosfogesso e $0,8 \%$ de ativador aos 28 dias de hidratação, percebe-se uma estrutura bem distinta da observada anteriormente (Figura 8). As agulhas de etringita são menores e a estrutura do CSH menos evidente. A imagem também sugere a formação de monossulfato, o qual seria devido à reação da etringita com íons remanescentes de cálcio e alumínio, observados em sistemas com baixos teores de sulfato de cálcio [20]. A imagem também sugere uma estrutura mais compactada que aquela exibida pela Figura 8, o que poderia justificar a maior resistência mecânica apresentada por esta pasta.
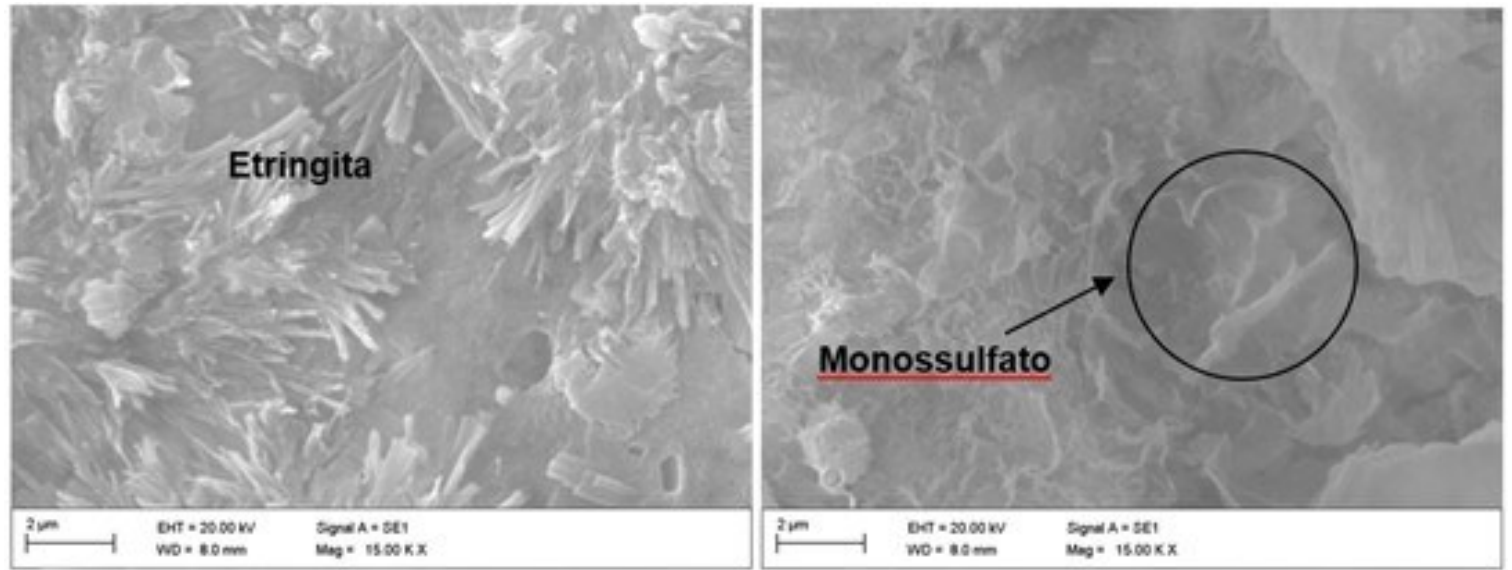

Figura 9: Microscopia eletrônica de varredura (MEV): Pasta 90_10 com 0,8\% de ativador, aos 28 dias 


\section{CONCLUSÕES}

Neste artigo o fosfogesso foi estudado como fonte alternativa de sulfato de cálcio em cimentos supersulfatados (CSS). A partir das análises, pode-se verificar que:

- Os valores de resistência à compressão das amostras de CSS apresentaram bom desempenho aos $7 \mathrm{e}$ 28 dias de hidratação. Todos as amostras alcançaram a resistência mínima exigida pela EN 15743, com exceção daquela contendo $10 \%$ de fosfogesso e ativador alcalino $0,2 \%$, cuja a resistência foi inferior a $32,5 \mathrm{MPa}$ aos 28 dias de hidratação;

- Os ensaios de calorimetria mostraram que as pastas apresentaram uma taxa de hidratação lenta, como picos pouco intensos em tempos mais longos, típicos em CSS, cuja cinética foi mais influenciada pelo teor de ativador alcalino do que pelo teor de sulfato de cálcio;

- As análises de DRX e DSC identificaram a presença de etringita como principal composto de hidratação, em todas as amostras. Em ambas as técnicas, a presença do CSH foi pouco evidente, devido a sua baixa cristalinidade, no caso do DRX, e da sua ocorrência próxima à etringita, no caso do DSC, mas sua presença foi confirmada no MEV;

- O excesso de ativador alcalino $(\mathrm{KOH})$ teve implicações distintas nas pastas com diferentes percentuais de sulfato de cálcio. Naquelas com $10 \%$, o alto teor $\mathrm{KOH}(0,8 \%)$ implicou queda de resistência e foi associada à intensa precipitação e crescimento de cristais de etringita na superfície dos grãos de escória, causando seu isolamento e inibindo posterior hidratação. Já, naquelas com $20 \%$ de sulfato de cálcio, o alto ter de $\mathrm{KOH}$ implicou a instabilidade da etringita, que neste estudo, foi associada à sua conversão em monossulfato, porém, porém mais investigações são necessárias para comprovar a hipótese. Investigações complementares, em idades iniciais e tardias, bem como avaliação da porosidade e expansão vêm sendo realizadas e serão objeto de futuras publicações.

- Cimento supersulfatado formado por $90 \%$ escória e $10 \%$ fosfogesso apresentou resistência mecânica superior a $35 \mathrm{MPa}$, mostrando uma alternativa ao cimento Portland relacionada, sobretudo, ao seu baixo impacto ambiental.

\section{AGRADECIMENTOS}

Os autores agradem ao CNPq pelo apoio financeiro (Edital Universal 2013, processo 483661/2013-9), pela bolsa de iniciação científica, e à CAPES, pela bolsa de mestrado.

\section{BIBLIOGRAFIA}

[1] JUENGER, M.C.G., WINNEFELD, F., PROVIS, J.L., et al., "Advances in alternative cementitious binders", Cemente and Concrete Research, v. 41, n. 12, pp.1232-1243, 2011.

[2] GRUSKOVNJAK, A., FIGI, R., LOTHENBACH, B., et al., "Quantification of hydration phases in supersulfated cements: review and new approaches", Advence in Cement Research,v. 23, n. 6, pp. 265-275, Jun. 2011.

[3] GROUNDS, Z., NOWELL, D. V., WILBURN, F.W., "The influence of temperature and different storage conditions on the stability of supersulphated cement", Journal of Thermal Analysis, v. 41, pp. 687-699, 1994.

[4] GROUNDS, Z., NOWELL, D. V., WILBURN, F.W., "Resistance of Supersulfated Cement To Strong Sulfate Solutions", Journal of Thermal Analysis and Calorimetry, v. 72, pp. 181-190, 2003.

[5] KÜHL, H., Verfahrenzur Herstellung von Zementaus Hochofenschlacke, German Patent, 1908.

[6] KÜHL, H,, SCHLEICHER E. Gipsschlackenzement, Fachbuchverlag GmbH, Leipzig, Germany, 1952.

[7] NOVAK, D., NOVAK, R., Practical experience with a new type of supersulfated cement, In: World Cement, 2004.

[8] DIN 4210. Sulfathüttenzement, Deutsches Institut für Normung. 19AD.

[9] EN 15743, Supersulfated cement - Composition, specifications and conformity criteria, 2010.

[10] ANGULSKI DA LUZ, C., HOOTON, R. D., "Influence of curing temperature on the process of hydration of supersulfated cements at early age", Cement and Concrete Research, v. 77, pp.69-75, Jul, 2015.

[11] GRUSKOVNJAK, A., LOTHENBACH, B., WINNEFELD, F., et al., "Hydration mechanisms of super sulphated slag cement", Cement and Concrete Research, v. 32, pp.983-992, Mar, 2008.

[12] RUBERT, S., Contribuição ao estudo de cimentos supersulfatados: formulação e mecanismos de hidratação, Dissertação de M.Sc., PPGTP/UTFPR, Pato Branco, PR, Brasil, 2015.

[13] CANUT, M. M. C., JACOMINO, V. M. F., BRÅTVEIT K, G. A., et al., "Microstructural analyses of phosphogypsum generated by Brazilian fertilizer industries", Materials Characterization, v. 59, pp.365-373, 
2008.

[14] SAUEIA, C. H. R., MAZZILLI, B. P., "Distribution of natural radionuclides in the production and use of phosphate fertilizers in Brazil”, Journal of Environmental Radioactivity, v. 89, pp. 2295-239, Mai. 2006.

[15] SCHAEFER, O. C., Valorização de Fonte alternativa de Sulfato de Cálcio para a produção de argamassas autonivelantes, Tese de D.Sc., PPGEC/UFSC, Florianópolis, SC, Brasil, 2013.

[16] CUADRI, A. A., NAVARRO, F. J., GARCÍA-MORALES, M., et al., "Valorization of phosphogypsum waste as asphaltic bitumen modifier”, Journal of Hazard Materials, v. 279, pp. 11-16, 2014.

[17] TAYIBI, H., CHOURA, M., LÓPEZ, F. A., et al., "Environmental impact and management of phosphogypsum”, Journal of Environmental Management, v. 90, pp. 2377-2386, Mar, 2009.

[18] OSSORIO, M., DRIESSCHE, A. E. S. V., PÉREZ, P., et al., "The gypsum - anhydrite paradox revisited", Chemical Geology, v. 386, pp. 16-21, 2014.

[19] SEUFERT, S., HESSE, C., GOETZ-NEUNHOEFFER, F., et al., "Quantitative determination of anhydrite III from dehydrated gypsum by XRD”, Cement and Concrete Research, v. 39, pp.936-941, Jun, 2009.

[20] MUN, K. J., HYOUNG, W. K., LEE, C. W., et al. "Basic properties of non-sintering cement using phosphogypsum and waste lime as activator", Construction and Building Materials, v. 21, pp.1342-50, 2007. [21] ERDEM, E., OLMEZ, H., "The mechanical properties of supersulphated cement containing phosphogypsum", Cement and Concrete Research, v. 23, pp.115-121, 1993.

[22] DUTTA, D. K., BORTHAKUR, P. C., “Activation of low high alumina graulated blast furnace slag by anydrite", Cement and Concrete Research, v. 20, pp.711-722, 1990.

[23] DING, S., SHUI, Z., CHEN, W., et al., "Properties of supersulphated phosphogysum slag cement (SSC) concrete", Journal Wuhan University of Technology, Materials Science Edition, v. 29, pp. 109-113, Fev. 2014.

[24] SINGH, M., GARG, M., "Calcium sulfate hemihydrate activated low heat sulfate resistant cement", Construction and Building Materials, v. 16, pp. 181-186, 2002.

[25] ERDEM E, OLMEZ H. "The mechanical properties of supersulphated cement containing phosphogypsum”, Cement and Concrete Research, v. 23, pp.115-121, 1993.

[26] GRACIOLI, B., VARELA, M.V.F., ANGULSKI DA LUZ, C. "Utilização de fosfogesso em matrizes de cimentos supersulfatados: um subproduto da indústria de fertilizantes”, In: III Congresso de Ciência e Tecnologia da UTFPR - DV, pp. 529-31, Dois Vizinhos, Out, 2015.

[27] GRACIOLI, B., VARELA, M. V. F., RUBERT, S., et al., "Valorization of phosphogypsum in Supersulfated Cement (SSC): a contribution for binders free of CO2 emissions", In: 16th NOCMAT 2015, Winnipeg, 2015.

[28] MATSCHEI, T., BELLMANN, F., STARK, J. "Hydration behaviour of sulphate-activated slag cements", Advances in Cement Research, v. 17, n. 4, p. 167-178, 2005.

[29] AMERICAN SOCIETY FOR TESTING AND MATERIALS - ASTM. Standard Specification for Compressive Strength of Hydraulic Cement Mortars (Using 2-in. or [50-mm] Cube Specimens), ASTM C109/C, West Conshohocken, PA, United States, 2008. 